\title{
Atrial Tachycardia With Widely-Split P Waves may Mimic a Distinct Faster Atrial Tachycardia With Half the Cycle Length of the Actual P-P Interval
}

\author{
Takumi Yamada, MD; Harish Doppalapudi, MD; \\ H. Thomas McElderry, MD; G. Neal Kay, MD
}

\begin{abstract}
A 57-year-old man with atrial tachycardia (AT) after cavo-tricuspid isthmus ablation underwent electrophysiological testing. Mapping revealed atrial activation with a cycle length exactly twice that of the P-P interval on electrocardiogram. Electroanatomic mapping revealed an AT focus near the left superior pulmonary vein, with inter-atrial conduction occurring in the lower septum and cavo-tricuspid isthmus conduction block. A propagation map demonstrated activation going down the left atrial posterior wall and right atrial free wall, generating different positive $\mathrm{P}$ waves in the inferior leads. The focal AT with inter- and intra-atrial conduction block exhibited widely-split $\mathrm{P}$ waves misinterpreted as a distinct AT. (Circ J 2008; 72: 1381-1384)
\end{abstract}

Key Words: Focal atrial tachycardia; Inter-atrial conduction disturbance; Radiofrequency catheter ablation; Split $\mathrm{P}$ wave

I t has been reported that intra- and/or inter-atrial conduction block can generate split $\mathrm{P}$ waves.,2 We report a case in which an atrial tachycardia (AT) with an intra- and inter-atrial conduction block exhibited widelysplit $\mathrm{P}$ waves that were misinterpreted as a distinct AT with half the cycle length (CL) of the original AT.

\section{Case Report}

A 57-year-old man with hypertension underwent catheter ablation for cavo-tricuspid isthmus dependent atrial flutter and subsequently developed another supraventricular tachycardia (SVT) for which he was referred for an electrophysiologic study (EPS) and catheter ablation. During sinus rhythm (SR), the 12-lead electrocardiogram (ECG) exhibited a split P wave with a duration of $190 \mathrm{~ms}$ (Fig 1). Written informed consent was given and the EPS was performed after all antiarrhythmic drugs had been discontinued for at least 5 half-lives prior to the study. At baseline, SVT persisted and during it the 12-lead ECG appeared to exhibit regular P waves (CL $150 \mathrm{~ms}$ ) (Fig 1). Two 6Fr multipolar catheters were introduced via the right common femoral vein and positioned in the coronary sinus (CS) and low lateral right atrium (RA) for mapping and pacing. Activation mapping revealed regular atrial activations with CLs of

(Received September 25, 2007; revised manuscript received December 19, 2007; accepted December 25, 2007)

Division of Cardiovascular Disease, University of Alabama at Birmingham, Birmingham, AL, USA

There was no financial support for this study.

Mailing address: Takumi Yamada, MD, PhD, Division of Cardiovascular Disease, University of Alabama at Birmingham, VH B147, 1670 University Boulevard, 1530 3rd AVE S, Birmingham, AL 35294-0019, USA. E-mail: takumi-y@ fb4.so-net.ne.jp

All rights are reserved to the Japanese Circulation Society. For permissions, please e-mail: cj@j-circ.or.jp
$300 \mathrm{~ms}$ in both atria, which were just twice as long as the P$\mathrm{P}$ interval (Fig 1). The atrial activations within the CS were recorded in the mid-diastolic period of the atrial activations at the low lateral RA. Rapid atrial pacing from the low lateral RA was then performed and revealed a very long postpacing interval (PPI) $(830 \mathrm{~ms})$ (Fig 2). During the first beat of the SVT immediately after the cessation of pacing, the earliest atrial activation was observed in the mid CS (Fig 2). Rapid atrial pacing from the CS revealed that the PPI in the mid CS $(450 \mathrm{~ms})$ was shorter than that in the proximal CS (480 ms) (Fig 3). After transseptal catheterization, electroanatomic mapping with a $7.5 \mathrm{Fr}, 3.5-\mathrm{mm}$ tip-irrigated ablation catheter (NAVI-STAR ${ }^{\mathrm{TM}}$ THERMOCOOL ${ }^{\mathrm{TM}}$, Biosense Webster, Diamond Bar, CA, USA) was then performed in the left atrium (LA) during SVT. It revealed a centrifugal activation pattern from the posterior wall near the left superior pulmonary vein (Fig 4). Following that, electroanatomic mapping in the RA was also performed during SVT and revealed a centrifugal activation pattern from the foramen ovale and CS ostium, and conduction block at the cavotricuspid isthmus produced by the previous catheter ablation of atrial flutter (Fig 4). A propagation map demonstrated that activations going down the LA posterior wall from the AT focus and down the RA free wall caused the split positive $\mathrm{P}$ waves in the inferior leads. Radiofrequency application with a target temperature of less than $40^{\circ} \mathrm{C}$ and power output titrated up to $40 \mathrm{~W}$ was delivered at the earliest atrial activation site and successfully eliminated the AT. Electroanatomic mapping in the LA was then performed during SR and revealed a centrifugal activation pattern, starting from the putative insertion site of Bachmann's bundle (Fig 4).

\section{Discussion}

It is often difficult to recognize the $\mathrm{P}$ waves during SVT when they override the $T$ waves. In the present case, the 


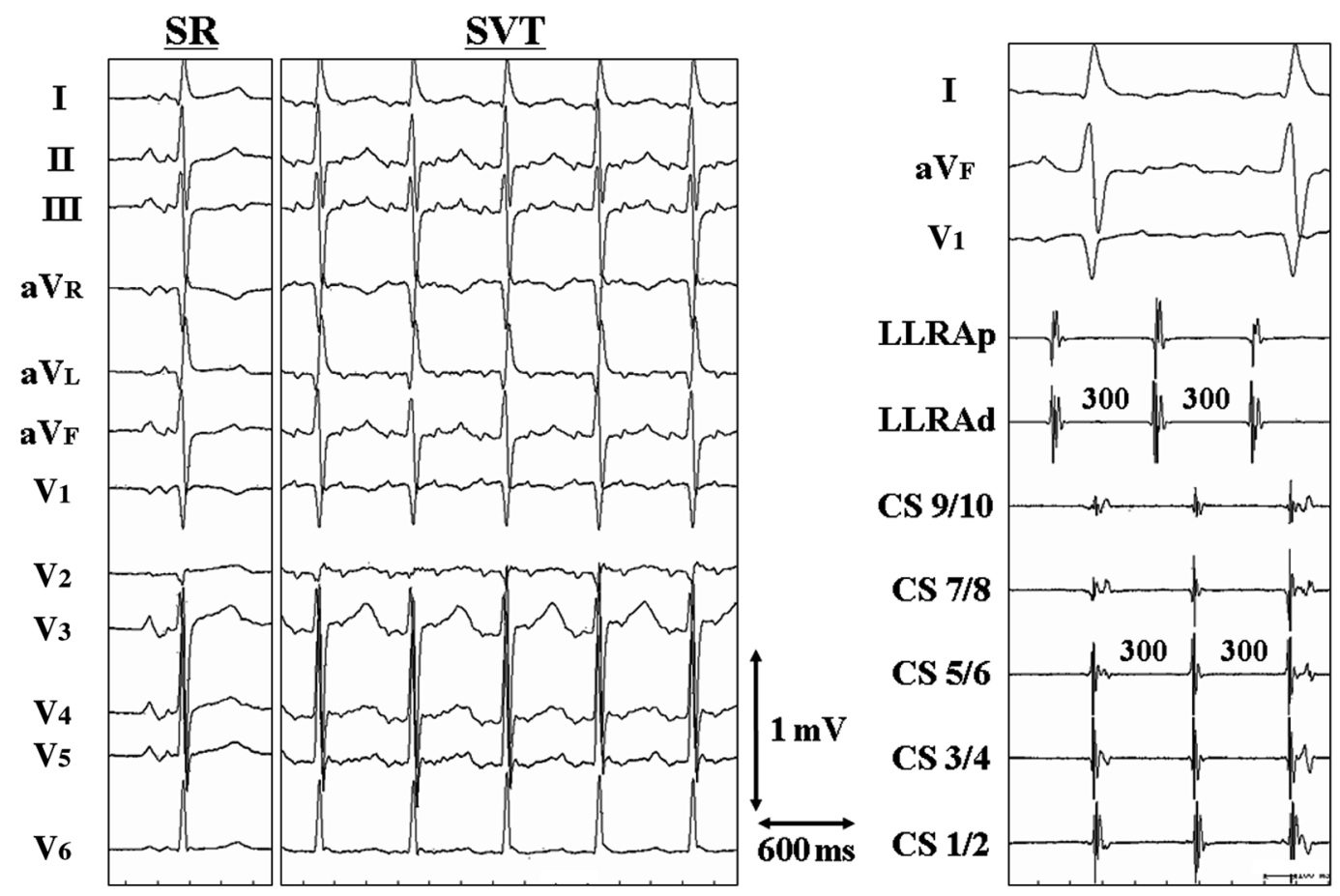

Fig 1. Twelve-lead electrocardiogram recordings during sinus rhythm (SR) (Left panel) and the supraventricular tachycardia (SVT) (Mid panel), and the cardiac tracings recorded during the SVT (Right panel). CS, coronary sinus; LLRA d,p, distal and proximal electrode pairs of the catheter positioned in the low lateral right atrium.

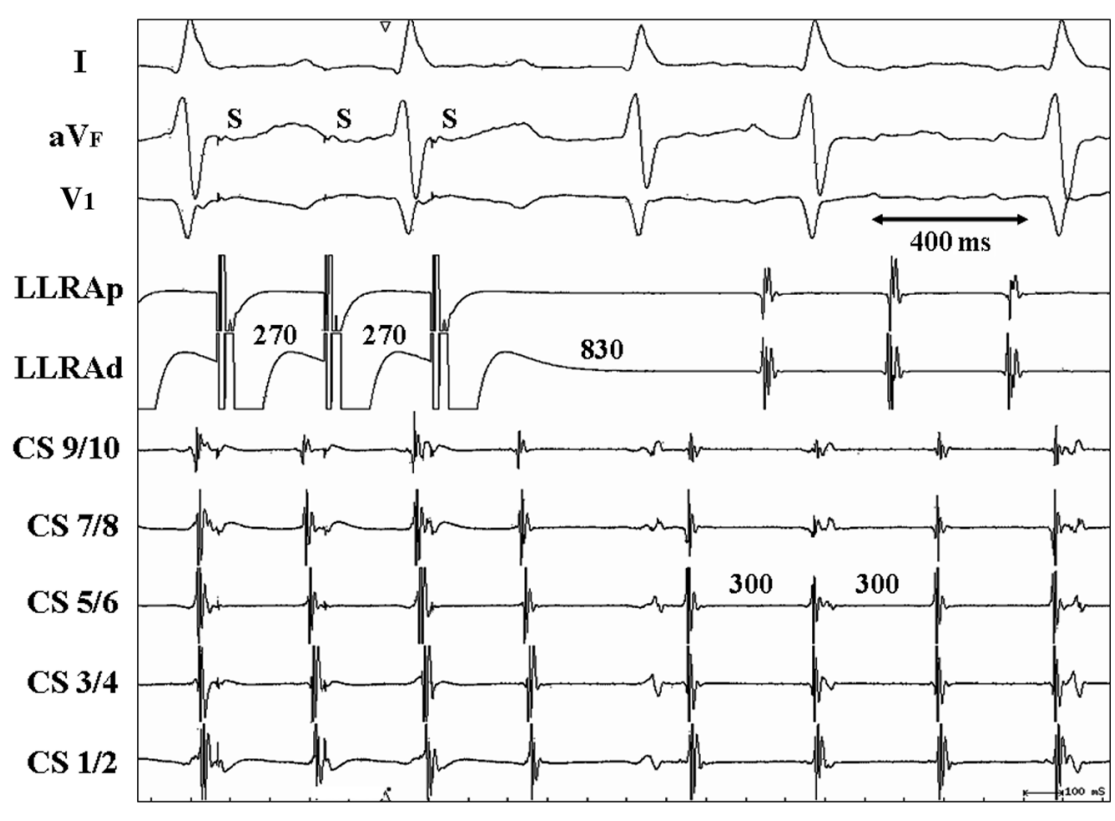

Fig 2. Cardiac tracings showing rapid atrial pacing from the low lateral right atrium during the tachycardia. S, stimulation. Other abbreviations as in Fig 1. intervals between the peaks or nadirs of the $\mathrm{P}$ waves in the inferior leads or lead $\mathrm{aV}_{\mathrm{L}}$ were perfectly regular, and the tachycardia mimicked a distinct AT with half the CL of the original AT. Careful observation might have revealed P-wave alternans in several leads, especially the right precordial leads, and those findings might have been an electrocardiographic clue for abandoning the wrong diagnosis of tachycardia. In this case, split $\mathrm{P}$ waves during SR suggested the existence of a potential inter-atrial conduction block ${ }^{2}$ however, such a consideration might not have led to the mechanism of this tachycardia before the EPS. During
SR in normal hearts, the activation from the RA conducts rapidly via Bachman's bundle to the LA. On the other hand, it has been demonstrated that activation from the LA conducts to the RA via Bachman's bundle, the foramen ovale or CS? In this case, activation from the RA conducted via Bachman's bundle to the LA during SR, though a conduction disturbance should have existed in Bachman's bundle during SR as the split P waves suggested. On the other hand, activation from the LA did not conduct to the RA via Bachman' bundle, but instead via the foramen ovale and $\mathrm{CS}$ ostium during the AT. Therefore, activation from the 


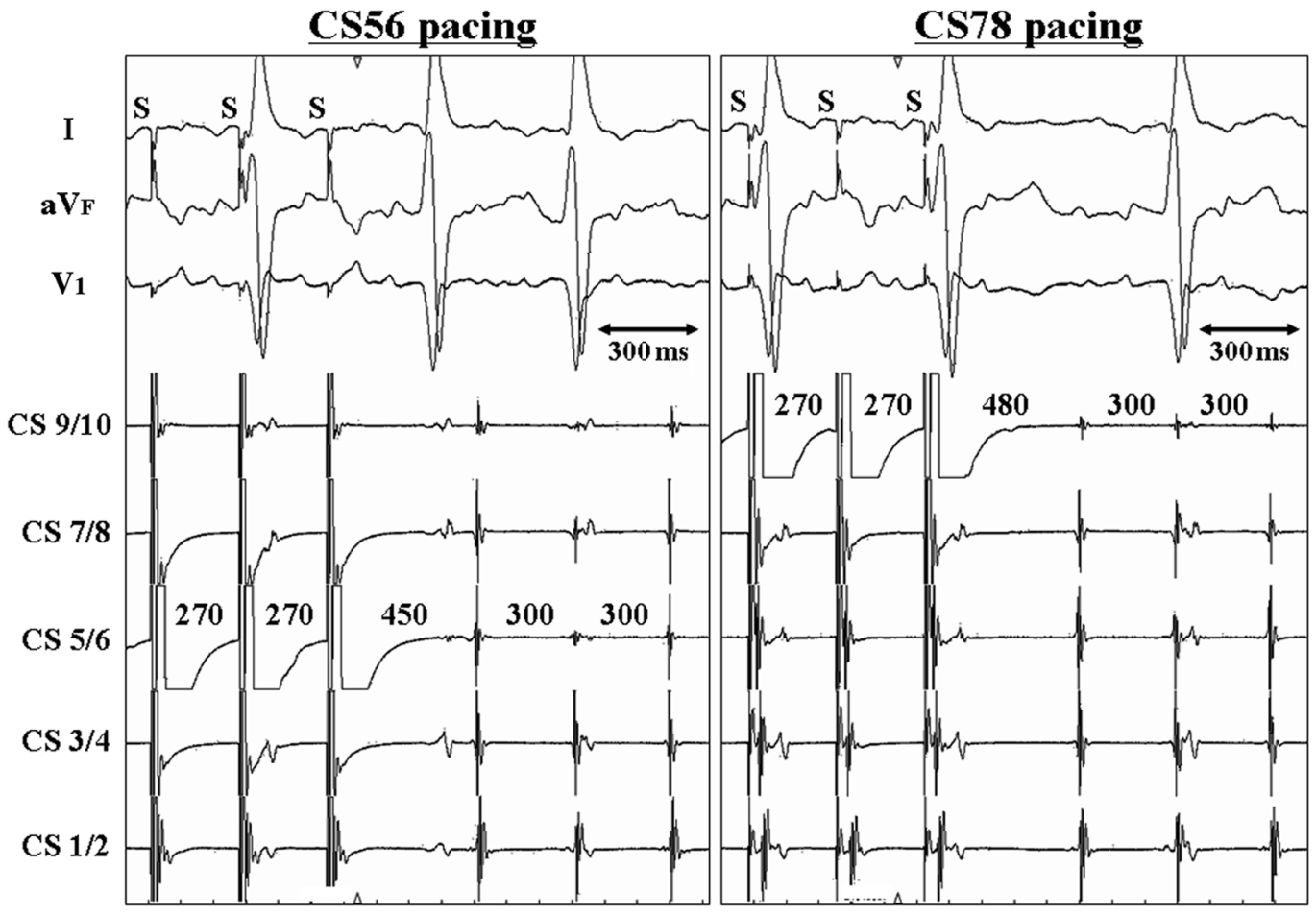

Fig 3. Cardiac tracings showing rapid atrial pacing from the CS during the tachycardia. Abbreviations as in Figs 1,2.
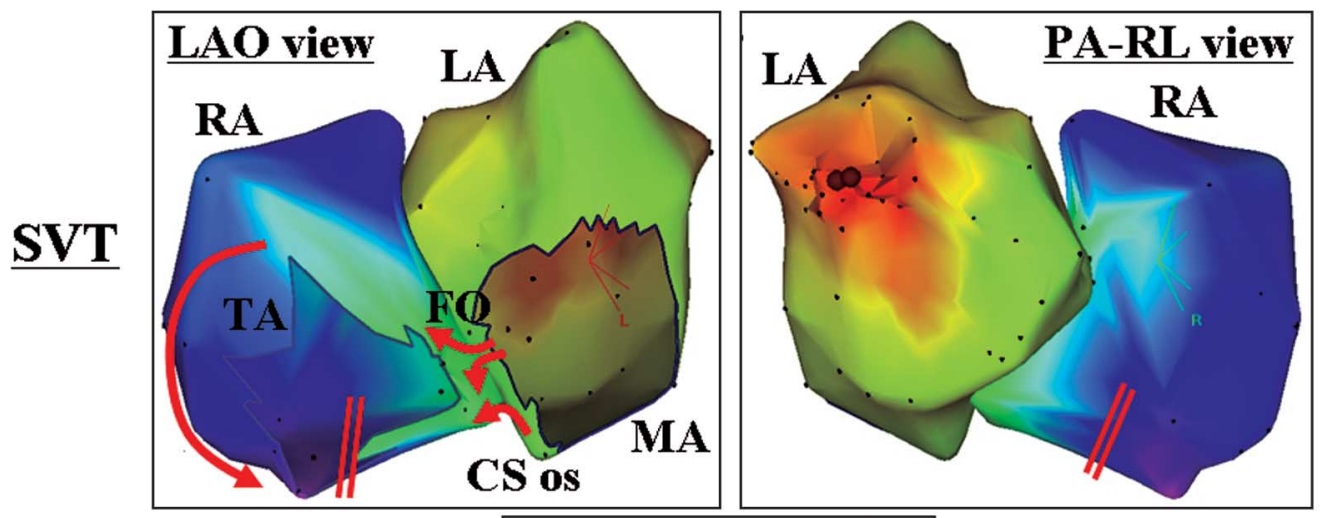

$202 \mathrm{~ms}$

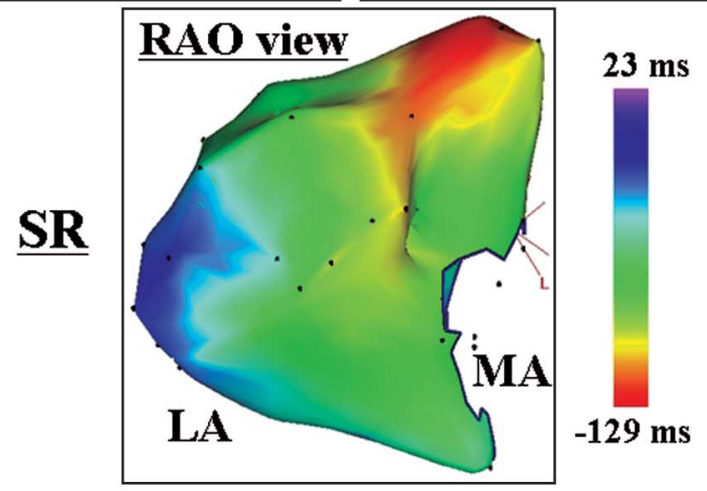

Fig 4. Electroanatomic maps of both atria during the tachycardia (Upper panels) and left atrium (LA) during sinus rhythm (Lower panel). The red indicates the areas with the earliest endocardial activation and orange, yellow, green, blue and purple indicate progressively delayed activation. The red arrow indicates the activation sequences and the double red lines the conduction block at the cavo-tricuspid isthmus. FO, foramen ovale; LAO, left anterior oblique; MA, mitral annulus; os, ostium; PA-RL, postero-anterior-right lateral; RA, right atrium; RAO, right anterior oblique; TA, tricuspid annulus. Other abbreviations as in Fig 1. 
AT focus near the left superior pulmonary vein propagated across the LA to that inter-atrial conduction site. Consequently, activation in the RA started after that in the LA had finished. Such an inter-atrial conduction pattern might produce a split $\mathrm{P}$ wave. Intra-atrial conduction block at the cavo-tricuspid isthmus also played a role in splitting the $\mathrm{P}$ wave more widely. This case demonstrated that focal AT with an inter- and intra-atrial conduction block might exhibit widely-split $\mathrm{P}$ waves that could be misinterpreted as a distinct AT with half the $\mathrm{CL}$ of the original AT.

\section{References}

1. Soejima K, Mitamura H, Miyazaki T, Akaishi M, Miyoshi S, Ogawa $\mathrm{S}$, et al. A case of widely split double $\mathrm{P}$ waves with marked intra-atrial conduction delay. J Cardiovasc Electrophysiol 1997; 8: 1296-1301.

2. Ariyarajah V, Asad N, Tandar A, Spodick DH. Interatrial block: Pandemic prevalence, significance, and diagnosis. Chest 2005; 128: 970-975.

3. Roithinger FX, Cheng J, SippensGroenewegen A, Lee RJ, Saxon LA, Scheinman MM, et al. Use of electroanatomic mapping to delineate transseptal atrial conduction in humans. Circulation 1999; 100: $1791-1797$. 